\title{
Solid part size is an important predictor of nodal metastasis in lung cancer with a subsolid tumor
}

\author{
Jun Yeun Cho ${ }^{1,2}$, Cho Sun Leem ${ }^{1}$, Youlim Kim ${ }^{1,2}$, Eun Sun Kim,2, Sang Hoon Lee ${ }^{1,2}$, Yeon Joo Lee ${ }^{1,2}$, \\ Jong Sun Park ${ }^{1,2}$, Young-Jae Cho ${ }^{1,2}$, Jae Ho Lee ${ }^{1,2}$, Choon-Taek Lee ${ }^{1,2}$ and Ho $\|$ Yoon ${ }^{1,2^{*}}$
}

\begin{abstract}
Background: Candidates for preoperative or intraoperative nodal assessment among patients with non-small cell lung cancer (NSCLC) manifesting as a subsolid tumor are not established. The present study was conducted to demonstrate the distribution of nodal metastasis rate according to newly proposed T categories for subsolid tumors, and we further aimed to identify radiologic parameters that can be predictive of nodal metastasis.

Methods: We retrospectively reviewed cases of NSCLC manifesting as a subsolid tumor in computed tomography scans in a university-affiliated tertiary hospital between April 2013 and August 2016. All patients underwent mediastinal lymph node dissection during resection surgery. Multivariate analysis was performed among clinical and radiologic parameters.

Results: Of the 269 eligible patients, T-categories were classified as cTis $(n=23,8.6 \%)$, cT1 $(n=203,75.5 \%)$, and cT2 $(n=43,16.0 \%)$. Ten patients (3.7\%) had nodal metastasis: pN1 $(n=5,1.9 \%)$, pN2 $(n=5,1.9 \%)$. Nodal metastasis was not observed in tumors with a solid part $\leq 1.0 \mathrm{~cm}$ ( $\mathrm{cT} 1 \mathrm{mi}$ and $\mathrm{CT} 1 \mathrm{a})$ or in nonsolid tumors $\leq 3.0 \mathrm{~cm}$ (cTis). The nodal metastasis rate in CT1 b, CT1c, and cT2 tumors was 6.1\% (4/65), 8.3\% (1/12), and 11.7\% (5/43), respectively. Multivariate analysis showed that a solid part size $>1.5 \mathrm{~cm}$ [odds ratio, 5.89; 95\% confidence interval, $1.25-27.68, p=0.025$ ] was significantly associated with nodal metastasis.

Conclusions: We observed nodal metastasis from cT1 b tumors (solid part size $>1 \mathrm{~cm}$ ) among proposed T categories for subsolid tumors and a solid part size is an important radiologic parameter predictive of nodal metastasis in NSCLC manifesting as a subsolid tumor. Considering the low rate of nodal metastasis, pathologic nodal assessment may be unnecessary in early $T$ category tumors with a small solid part size.
\end{abstract}

Keywords: Risk factor, Non-small cell lung Cancer, Lymphatic metastasis

\section{Background}

The considerable increase in lung cancer screening has recently led to issues such as an increased number of lung nodules discovered via computed tomography (CT) and the management of these lesions. The subsolid nodule, defined as a well-demarcated lung lesion containing a ground-glass opacity, exhibits different behaviors from a solid nodule and accordingly, has garnered much

\footnotetext{
*Correspondence: dextro70@gmail.com

${ }^{1}$ Division of Pulmonary and Critical Care Medicine, Department of Internal Medicine, Seoul National University Bundang Hospital, 82, Gumi-ro 173 Beon-gil, Bundang-gu, Seongnam-si, Gyeonggi-do 13620, Republic of Korea ${ }^{2}$ Department of Internal Medicine, Seoul National University College of Medicine, Seoul, Republic of Korea
}

attention. In 2013, the Fleischner Society recommendations emphasized that from a management perspective, both pure and part-solid ground-glass nodules are best considered as a category separate from purely solid lesions [1]. Subsequently, many studies have investigated subsolid nodules, and consequently, the recently proposed eighth tumor-node-metastasis staging system includes more detailed T1 categories of subsolid nodules, compared to previous versions [2].

Accurate nodal staging is fundamental in diagnosing and treating non-small cell lung cancer (NSCLC). Generally, the nodal metastasis rate in patients with NSCLC manifesting as a subsolid nodule has been known to be 
low [3-5]. Therefore, it is questionable whether preoperative or intraoperative nodal assessment is needed. Recently updated National Comprehensive Cancer Network guidelines state that preoperative pathologic mediastinal evaluation is optional for solid tumors $<1.0 \mathrm{~cm}$ and purely non-solid tumors $<3.0 \mathrm{~cm}$ with radiologic negative mediastinum [6] because of the low rate of mediastinal metastasis. Intraoperative nodal assessment in this situation is also controversial, and there is no consensus. Currently, many clinicians conduct intraoperative nodal assessment based on their experiences.

Given this background, the present study was conducted to demonstrate the distribution of nodal metastasis rate according to newly proposed $\mathrm{T}$ categories for subsolid tumors. For selecting candidates for preoperative or intraoperative nodal assessment, we further aimed to identify radiologic parameters that can be predictive of nodal metastasis.

\section{Methods}

We selected patients with surgically resected NSCLC manifesting as a subsolid tumor on CT scans in a university affiliated-tertiary hospital from April 2013 to August 2016. All patients underwent mediastinal lymph node dissection (MLND) during resection surgery. The patient's clinical data, radiologic features, and pathologic results were retrospectively reviewed.

The definitions of nodal zone and nodal station were based on the International Association of the Study of Lung Cancer (IASLC) lymph node map [7]. The pathologic diagnoses were based on the 2011 IASLC classification [8]. Real-time endobronchial ultrasound (EBUS) was performed in selected patients. Rapid on-site evaluation was not conducted during EBUS procedure.

We described radiologic features of a subsolid tumor based on expert thoracic radiologists' reports. In the present study, we defined a subsolid tumor as a mass (> $3 \mathrm{~cm})$ or nodule $(\leq 3 \mathrm{~cm})$ that contained ground-glass lesions on CT images. The total tumor (including ground glass portion around solid part) and solid part size were measured as the maximum diameters on the lung window setting. Subsolid tumors less than or equal to $3 \mathrm{~cm}$ were classified according to recently proposed $\mathrm{T}$ categories [2].T categories were classified by the total tumor and solid part size; cTis (total tumor size of $0.6-3.0 \mathrm{~cm}$ with no solid part), cT1 (total tumor size of $0.6-3.0 \mathrm{~cm}$ with solid part size $0.6-3.0 \mathrm{~cm}$ ) and $\mathrm{cT} 2$ (total tumor size of 3.0-7.0 cm with any solid part size).

If a total tumor measured $3 \mathrm{~cm}$ to $7 \mathrm{~cm}$, it was categorized as CT2 regardless of the solid part size. Cases with multiple tumors were excluded. A tumor was considered central if it was visualized within the inner third of the lung field or abutted mediastinal structures on CT or positron emission tomography (PET) images. Radiologic
$\mathrm{N}$ staging was determined from CT scans with or without PET findings. Lymph nodes with shortest diameters of $>1 \mathrm{~cm}$ on $\mathrm{CT}$ and/or a maximum standardized uptake value $>2.5$ on PET were considered metastatic. Pathologic $\mathrm{N}$ stage was determined by the final pathologic report after surgery.

All data are presented as mean values ( \pm standard deviations) for continuous variables and numbers (percentages) for categorical variables. Data were compared between defined groups using the Mann-Whitney test for continuous variables and Fisher's exact test for categorical variables. A linear-by-linear association was defined using Pearson's

Table 1 Clinical, radiologic and pathologic characteristics of patients

\begin{tabular}{ll}
\hline Characteristic & Total $(n=269)$ \\
\hline Age, years & $62.4 \pm 10.4$ \\
Male sex & $115(42.8)$ \\
Former/Current smoker & $90(33.5)$ \\
Previous extra-thoracic malignancy & $34(12.6)$ \\
Previous lung cancer & $6(2.2)$ \\
Tumor centrality & $14(5.2)$ \\
Radiologic N stage & \\
NO & $227(84.4)$ \\
N1-2 & $42(15.6)$ \\
T categories for subsolid tumor ${ }^{\mathrm{a}}$ & \\
CTis & $23(8.6)$ \\
CT1 & $203(75.5)$ \\
cT2 & $43(16.0)$ \\
EBUS & $99(36.8)$ \\
Operation extent & \\
Segmentectomy & \\
Lobectomy & $41(15.2)$ \\
Pathology & $228(84.8)$ \\
AAH/AIS & $259(96.3)$ \\
MIA & $5(1.9)$ \\
Invasive ADC & $5(1.9)$ \\
Nathologic N stage ${ }^{b}$ &
\end{tabular}

Data are presented as $\mathrm{n}(\%)$ or mean \pm standard deviation

$A A H$ atypical adenomatous hyperplasia, $A D C$ adenocarcinoma, $A / S$ adenocarcinoma in situ, EBUS endobronchial ultrasound, MIA minimally invasive adenocarcinoma

${ }^{\mathrm{a}} \mathrm{T}$ categories were classified by the total tumor size $(\mathrm{cm})$ and solid part size $(\mathrm{cm})$. Total tumor size was defined as a size including ground glass around the solid part; cTis (total tumor size of $0.6-3.0 \mathrm{~cm}$ with no solid part), cT1 (total tumor size of $0.6-3.0 \mathrm{~cm}$ with solid part size $0.6-3.0 \mathrm{~cm}$ ) and cT2 (total tumor size of $3.0-7.0 \mathrm{~cm}$ with any solid part size)

${ }^{b}$ Pathologic $\mathrm{N}$ stage was determined by the final pathologic report after surgery 
coefficient and performed to test the trends of ordinal scales in categorical variables.

Significant variables from clinical and radiologic data identified in the univariate analysis were used for multivariate analysis to elucidate predictive factors of nodal metastasis. SPSS 22.0 (IBM, Armonk, NY, USA) was used for the statistical analyses. A $p$ value $<0.05$ was considered statistically significant.

\section{Results}

A total of 269 eligible patients were identified, and their baseline characteristics are summarized in Table 1 . The patients had a mean age of $62.4 \pm 10.4$ years. One hundred fifteen patients $(42.8 \%)$ were male, and 90 (33.5\%) were former or current smokers. Fourteen (5.2\%) patients had a centrally located tumor. The tumors were categorized into three groups: cTis $(n=25,8.9 \%)$, cT1 $(n=212,75.7 \%)$, and cT2 $(n=43,15.4 \%)$. There were no cases presenting as a nonsolid tumor less than $0.5 \mathrm{~cm}$. Ninety-nine $(36.8 \%)$ patients underwent EBUS prior to resection surgery, and 228 patients $(84.8 \%)$ underwent lobectomy. The most frequent tissue pathology was invasive adenocarcinoma $(n=235,84.8 \%)$. We identified 10 patients with nodal metastasis: pN1 $(n=5,1.9 \%)$ and pN2 $(n=5,1.9 \%)$.

Patients with more advanced clinical $\mathrm{T}$ category frequently underwent EBUS and lobectomy (Table 2). In cT1 tumors, the $\mathrm{pN} 1$ and $\mathrm{pN} 2$ rates were 1.5 and $1.0 \%$, respectively, whereas there was no nodal metastasis in cTis tumors. In cT2 tumors, the $\mathrm{pN} 1$ and $\mathrm{N} 2$ rates were 4.7 and $7.0 \%$, respectively. More advanced $\mathrm{T}$ tumors had significantly more advanced nodal metastasis. In addition, invasive adenocarcinomas were frequently diagnosed in advanced $\mathrm{T}$ tumors.

The distribution of nodal metastasis in tumors classified by the proposed clinical $\mathrm{T}$ categories is detailed in Table 3. Nodal metastasis was observed in tumors in which the solid part was $>1.0 \mathrm{~cm}$ or total tumor size $>$ $3.0 \mathrm{~cm}$. There was no nodal metastasis in cTis, cT1mi, and cT1a tumors. The nodal metastasis rates in cT1b, cT1c, and cT2 tumors were $6.1 \%(4 / 65), 8.3 \%(1 / 12)$, and $11.7 \%(5 / 43)$, respectively. The mean solid part size in cT2 tumors ( 2 cases were nonsolid tumors) was $2.0 \pm$ $1.0 \mathrm{~cm}$.

The detailed information of patients with nodal metastasis is presented in Table 4. All patients had a solid part exceeding $1 \mathrm{~cm}$. Only one patient had a centrally located tumor. Most cases of metastatic N1 involved a peribronchial node, and 3 of 5 cases of metastatic N2 involved a subcarinal node. Tumors with nodal metastasis were invasive adenocarcinoma with acinar, papillary, or solid subtype. Four patients with pN2 underwent EBUS prior to surgery. One patient (patient number 9) was diagnosed with pN2 disease by EBUS, and subsequently underwent surgery due to single N2. Other cases (patient number 7, 8, 10) were diagnosed with $\mathrm{pN} 2$ disease by surgery.

We performed a multivariate analysis to identify the risk factors predictive of nodal metastasis (Table 5). The multivariate analysis included variables that were identified as significant in the univariate analysis. A solid part

Table 2 Differences between cTis, CT1 and cT2 tumors

\begin{tabular}{|c|c|c|c|c|}
\hline Variables & cTis $(n=23)$ & CT1 $(n=203)$ & cT2 $(n=43)$ & $p$ Value \\
\hline EBUS & & & & 0.000 \\
\hline Not performed & $21(91.3)$ & $134(66.0)$ & $15(34.9)$ & \\
\hline Performed & $2(8.7)$ & $69(34.0)$ & $28(65.1)$ & \\
\hline Operation extent & & & & 0.009 \\
\hline Segmentectomy & $5(21.7)$ & $36(17.7)$ & $0(0.0)$ & \\
\hline Lobectomy & $18(78.3)$ & $167(82.3)$ & $43(100.0)$ & \\
\hline Pathologic N stage & & & & 0.039 \\
\hline No & $23(100.0)$ & $198(97.5)$ & $38(88.4)$ & \\
\hline N1 & $0(0.0)$ & $3(1.5)$ & $2(4.7)$ & \\
\hline N2 & $0(0.0)$ & $2(1.0)$ & $3(7.0)$ & \\
\hline Pathology & & & & 0.002 \\
\hline $\mathrm{AAH} / \mathrm{AIS}$ & $0(0.0)$ & $2(1.0)$ & $0(0.0)$ & \\
\hline MIA & $7(30.4)$ & $22(10.8)$ & $0(0.0)$ & \\
\hline Invasive ADC & $16(69.6)$ & $176(86.7)$ & $43(100.0)$ & \\
\hline Invasive mucinous ADC & $0(0.0)$ & $3(1.5)$ & $0(0.0)$ & \\
\hline
\end{tabular}

Data are presented as $\mathrm{n}(\%)$

$A A H$ atypical adenomatous hyperplasia, $A D C$ adenocarcinoma, AIS adenocarcinoma in situ, EBUS endobronchial ultrasound, MIA minimally invasive adenocarcinoma 
Table 3 Distribution of nodal metastasis in tumors classified by proposed clinical T categories ${ }^{a}$

\begin{tabular}{|c|c|c|c|c|c|}
\hline Categories & Total tumor Size ${ }^{\mathrm{b}}(\mathrm{cm})$ & Solid part Size $(\mathrm{cm})$ & $\mathrm{pNO}$ & $\mathrm{pN1}$ & pN2 \\
\hline cTis & $0.6-3.0$ & 0 & $23(100.0)$ & $0(0.0)$ & $0(0.0)$ \\
\hline cT1mi & $\leq 3.0$ & $\leq 0.5$ & $46(100.0)$ & $0(0.0)$ & $0(0.0)$ \\
\hline cT1a & $0.6-3.0$ & $0.6-1.0$ & $80(100.0)$ & $0(0.0)$ & $0(0.0)$ \\
\hline $\mathrm{cT} 1 \mathrm{~b}$ & $1.1-3.0$ & $1.1-2.0$ & $61(93.8)$ & $3(4.6)$ & $1(1.5)$ \\
\hline CT1C & $2.1-3.0$ & $2.1-3.0$ & $11(91.7)$ & $0(0.0)$ & $1(8.3)$ \\
\hline $\mathrm{CT}^{\mathrm{c}}$ & $3.0-7.0$ & Any & $38(88.4)$ & $2(4.7)$ & $3(7.0)$ \\
\hline
\end{tabular}

${ }^{a}$ The nodal metastasis rate was significantly different among classified T categories $(P=0.033)$

${ }^{b}$ Defined as a size including ground glass portion around the solid part

'Two cases were nonsolid tumors, and the mean solid part size was $2.0 \pm 1.0 \mathrm{~cm}$

size $>1.5 \mathrm{~cm}$ [odds ratio (OR), 5.89; 95\% confidence interval (CI), 1.25-27.68, $p=0.025$ ] was found to associate significantly with nodal metastasis.

\section{Discussion}

In our cohort, the nodal metastasis rate was $3.7 \%(1.9 \%$ with both $\mathrm{pN} 1$ and $\mathrm{pN} 2$ ). Among part-solid nodules (cT1a-cT1c), the $\mathrm{pN} 1$ and $\mathrm{pN} 2$ rates were 1.5 and $1.0 \%$, respectively, whereas there was no nodal metastasis in cTis (nonsolid nodule $\leq 3 \mathrm{~cm}$ ). These findings of low incidence of nodal metastasis are consistent with previous studies $[4,5,9]$.

When tumors were classified by the proposed new $\mathrm{T}$ categories, any nodal metastasis was observed from cT1b to cT2 tumors, in which the solid part size was > $1.0 \mathrm{~cm}$ or total tumor size $>3.0 \mathrm{~cm}$. In the final pathologic reports, patients with nodal metastasis had adenocarcinoma with invasive subtypes. There was no early stage adenocarcinoma (e.g., adenocarcinoma in situ (AIS), minimally invasive adenocarcinoma (MIA), lepidic predominant adenocarcinoma (LPA)). These findings suggest that preoperative pathologic nodal evaluation or extended lymph node assessment might be unnecessary when early stage adenocarcinomas are suspected by radiologic features.

It is debatable whether extended lymph node assessment should be performed in patients with early stage NSCLC. As noted earlier, the incidence of nodal metastasis in NSCLCs manifesting as a subsolid tumor is lower than in NSCLCs with pure solid tumor. Furthermore, the extent of intraoperative node assessment (e.g., selective sampling vs. systematic dissection) in NSCLC patients remains controversial [10]. Although more extended node dissection has positive effects on clinical outcomes [11, 12], it is important to consider various procedure-related morbidities. Flores et al. compared 151 cases of NSCLC (subsolid nodules) with MLND and 52 cases of NSCLC without MLND [13]. They observed no differences in survival and asserted that performing MLND is not mandatory when screen-diagnosed NSCLC manifests as a subsolid nodule.

Currently available guidelines recommend that preoperative pathologic mediastinal evaluation (e.g., EBUS) should be considered regarding tumor size, radiologic $\mathrm{N}$ status, and tumor centrality $[14,15]$. However, they do not suggest recommendations exclusively for subsolid tumors. In the

Table 4 Detailed characteristics of patients with nodal involvement ${ }^{a}$

\begin{tabular}{llllllllllll}
\hline $\begin{array}{l}\text { Patient } \\
\text { number }\end{array}$ & $\begin{array}{l}\text { Total } \\
\text { tumor size }\end{array}$ & $\begin{array}{l}\text { Solid } \\
\text { part size }\end{array}$ & T stage & Central Tumor & N Stage (CT) & N Stage (PET) & EBUS & pN stage & Involved N1 & $\begin{array}{l}\text { Involved N2 } \\
\text { Pathology/ } \\
\text { Subtype }\end{array}$ \\
\hline 1 & 3.0 & 1.7 & CT1b & No & 0 & 0 & Yes & pN1 & Interlobar & - & ADC/papillary \\
2 & 1.8 & 1.4 & CT1b & No & 0 & 0 & Yes & pN1 & Peribronchial & - & ADC/solid \\
3 & 4.1 & 2.3 & CT2 & No & 0 & 2 & Yes & pN1 & Peribronchial & - & ADC/acinar \\
4 & 3.2 & 3.1 & CT2 & No & 0 & 0 & Yes & pN1 & Peribronchial & - & ADC/papillary \\
5 & 2.7 & 1.2 & CT1b & No & 0 & 0 & Yes & pN1 & Peribronchial & - & ADC/acinar \\
6 & 3.7 & 2.8 & CT2 & No & 0 & 0 & No & pN2 & Peribronchial & $2,3,4$ & ADC/papillary \\
7 & 3.2 & 1.3 & CT2 & Yes & 0 & 0 & Yes & pN2 & - & 6 & ADC/acinar \\
8 & 5.4 & 2.0 & CT2 & No & 0 & 2 & Yes & pN2 & Peribronchial & 7 & ADC/acinar \\
9 & 2.7 & 2.6 & CT1c & No & 1 & 0 & Yes & pN2 & Peribronchial & 7 & ADC/acinar \\
10 & 2.4 & 1.8 & CT1b & No & 0 & 0 & Yes & pN2 & - & 7 & ADC/papillary \\
\hline ADC
\end{tabular}

$A D C$ adenocarcinoma, $C T$ computed tomography, EBUS endobronchial ultrasound, $P E T$ positron emission tomography

${ }^{\mathrm{a}}$ Four patients with pN2 underwent EBUS prior to surgery. One patient (patient number 9) was diagnosed with pN2 disease by EBUS, and subsequently

underwent surgery due to single N2. Other cases (patient number $7,8,10$ ) were diagnosed with pN2 disease by surgery 
Table 5 Risk factors predictive of nodal metastasis

\begin{tabular}{|c|c|c|c|c|c|}
\hline \multirow[b]{2}{*}{ Variables } & \multicolumn{2}{|c|}{ Univariate } & \multicolumn{3}{|c|}{ Multivariate } \\
\hline & $\mathrm{OR}$ & $95 \% \mathrm{Cl}$ & $\mathrm{OR}$ & $95 \% \mathrm{Cl}$ & $p$ Value \\
\hline Total tumor size $>3 \mathrm{~cm}$ & 5.81 & $1.61-21.05$ & 2.46 & $0.57-10.50$ & 0.226 \\
\hline Solid part size $>1.5 \mathrm{~cm}$ & 8.66 & $2.17-34.57$ & 5.89 & $1.25-27.68$ & 0.025 \\
\hline Tumor centrality & 2.10 & $0.25-17.87$ & & & \\
\hline Radiologic nodal metastasis & 2.42 & $0.60-9.75$ & & & \\
\hline
\end{tabular}

$\mathrm{Cl}$ confidence interval, $\mathrm{OR}$ odds ratio

present study, multivariate regression analysis showed that solid part size $(>1.5 \mathrm{~cm})$ is predictive of nodal metastasis. Previous studies focused on importance of solid consistency for predicting nodal metastasis in NSCLCs. Koike et al. suggested $89 \%$ solid consistency (proportion of solid part size in total tumor size including ground glass) as a cutoff value to predict mediastinal metastasis in clinical IA NSCLCs [16]. Gao et al. reported that an occult N2 risk was lower in tumors with a ground glass component than in tumors without, among T1-2 N0 NSCLCs determined by PET CT [17]. Ye et al. asserted that ground glass status (part solid or pure solid vs nonsolid) is more accurate predictor than tumor diameter in clinical IA adenocarcinoma [4]. However, these studies included numbers of pure solid tumors, not only subsolid tumors. Our study's strength is that only cases of NSCLC manifesting as subsolid tumors were included. This is an important finding because solid part size can be a determinant radiologic criterion when preoperative pathologic nodal assessments are performed.

Tumor size has been considered an important risk factor for predicting nodal metastasis, and preoperative pathologic nodal assessment was considered in cases of tumor exceeding 3 because of low negative predictive value of PET-CT for detecting mediastinal nodal metastasis $[18,19]$. However, total tumor size including ground glass was not a significant predictive factor (Table 5). The relatively small number of cT2 cases might have contributed to this result. Moreover, data from Table 2 shows that the nodal metastasis rate was significantly higher in cT2 tumors than in cTis or cT1 tumors. We believe that total tumor size is still worthy as an important predictive radiologic factor, as well as solid part size.

In our study, we did not find an association between tumor centrality and nodal metastasis, although tumor location has been considered an important factor related to NSCLC. In a study by Lee et al. [20] of 221 patients with clinical IA NSCLC with a radiologically negative mediastinum, the frequency of central tumor location was $23 \%$, with a higher incidence of $\mathrm{pN} 2$ disease relative to peripherally located tumors. However, only $5.2 \%$ of tumors in the present study were centrally located, and primary lung adenocarcinomas were generally located peripherally. Moreover, the term "tumor centrality" is vague even among radiologists. Hence, the ability of tumor centrality to predict nodal metastasis should be evaluated in future studies.

There are some limitations in our study. First, because of the retrospective design nature, selection bias may have affected the results. EBUS and lobectomy were performed more frequently in more advanced $\mathrm{T}$ categories (Table 2). Therefore, it is assumed that clinicians did not actively conduct preoperative or intraoperative nodal assessment in patients with early stage NSCLCs (AIS, MIA, and LPA). Indeed, we identified 376 surgically resected NSCLCs manifesting as a subsolid tumor during the study period and excluded 107 patients who did not undergo MLND. Second, the actual nodal metastasis rate was low because we included only subsolid tumors. Hence, logistic regression analysis showed widened confidence intervals for the odds ratios, subsequently indicating statistically weak data. Future studies correcting these limitations are needed.

\section{Conclusions}

Among the proposed $\mathrm{T}$ categories for subsolid nodules, we observed nodal metastasis from cT1b, in which the solid part size exceeded $1 \mathrm{~cm}$. The nodal metastasis rate was $3.7 \%$, and solid part size is an important radiologic parameter predictive of nodal metastasis in NSCLC manifesting as a subsolid tumor. Considering the low rate of nodal metastasis, pathologic nodal assessment may be unnecessary in early T category tumors with a small solid part size.

\section{Abbreviations \\ AIS: Adenocarcinoma in situ; Cl: Confidence interval; CT: Computed tomography; EBUS: Endobronchial ultrasound; IASLC: International Association of the Study of Lung Cancer; LPA: Lepidic predominant adenocarcinoma; MIA: Minimally invasive adenocarcinoma; MLND: Mediastinal lymph node dissection; NSCLC: Non-small cell lung cancer; OR: Odds ratio; PET: Positron emission tomography}

\section{Acknowledgements}

The authors would like to thank all thoracic surgery staffs at Seoul National University Bundang Hospital for their contributions to lung cancer surgery.

Funding

There is no funding supports for this study.

Availability of data and materials

The datasets used during the current study are available from the corresponding author on reasonable request. 


\section{Authors' contributions}

JYC, HIY were involved in the design of this study; JYC, HIY, CSL, YLK, ESK were involved in participant recruitment and data collection; SHL, YJL, JSP, YJC were involved in sample processing; JYC, JHL, CTL, HIY were all involved in manuscript preparation and editing. All authors approved the final manuscript.

\section{Ethics approval and consent to participate}

The need for informed consent for this study was waived due to the retrospective study design. The study was approved by the Institutional Review Board (IRB) and Ethics Committee of Seoul University Bundang hospital (IRB No. B-1611-372-105). This study was conducted in compliance with the Declaration of Helsinki.

\section{Competing interests}

All authors declare that they have no competing interests.

\section{Publisher's Note}

Springer Nature remains neutral with regard to jurisdictional claims in published maps and institutional affiliations.

Received: 28 May 2018 Accepted: 14 August 2018

Published online: 10 September 2018

\section{References}

1. Naidich DP, Bankier AA, MacMahon $\mathrm{H}$, et al. Recommendations for the management of subsolid pulmonary nodules detected at CT: a statement from the Fleischner society. Radiology. 2013;266:304-17.

2. Travis WD, Asamura H, Bankier AA, et al. The IASLC lung Cancer staging project: proposals for coding $T$ categories for subsolid nodules and assessment of tumor size in part-solid tumors in the forthcoming eighth edition of the TNM classification of lung cancer. J Thorac Oncol. 2016;11: 1204-23.

3. Hattori A, Suzuki K, Matsunaga T, et al. Is limited resection appropriate for radiologically "solid" tumors in small lung cancers? Ann Thorac Surg. 2012; 94:212-5.

4. Ye B, Cheng M, Li W, et al. Predictive factors for lymph node metastasis in clinical stage IA lung adenocarcinoma. Ann Thorac Surg. 2014;98:217-23.

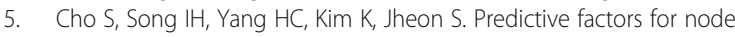
metastasis in patients with clinical stage I non-small cell lung cancer. Ann Thorac Surg. 2013;96:239-45.

6. Ettinger DS, Wood DE, Aisner DL, et al. Non-small cell lung Cancer, version 5. 2017, NCCN clinical practice guidelines in oncology. J Natl Compr Cancer Netw. 2017;15:504-35.

7. Rusch W, Asamura $\mathrm{H}$, Watanabe $\mathrm{H}$, et al. The IASLC lung cancer staging project: a proposal for a new international lymph node map in the forthcoming seventh edition of the TNM classification for lung cancer. J Thorac Oncol. 2009;4:568-77.

8. Travis WD, Brambilla E, Noguchi M, et al. American Thoracic Society. International Association for the Study of Lung Cancer/American Thoracic Society/European Respiratory Society: international multidisciplinary classification of lung adenocarcinoma: executive summary. Proc Am Thorac Soc. 2011;8:381-5.

9. Lee SM, Park CM, Paeng JC, et al. Accuracy and predictive features of FDGPET/CT and CT for diagnosis of lymph node metastasis of T1 non-small-cell lung cancer manifesting as a subsolid nodule. Eur Radiol. 2012;22:1556-63.

10. Lardinois D, De Leyn $P$, Van Schil $P$, et al. ESTS guidelines for intraoperative lymph node staging in non-small cell lung cancer. Eur J Cardiothorac Surg. 2006;30:787-92.

11. Lardinois D, Suter H, Hakki H, Rousson V, Betticher D, Ris H-B. Morbidity, survival, and site of recurrence after mediastinal lymph-node dissection versus systematic sampling after complete resection for non-small cell lung cancer. Ann Thorac Surg. 2005;80:268-75.

12. Doddoli C, Aragon A, Barlesi F, et al. Does the extent of lymph node dissection influence outcome in patients with stage I non-small-cell lung cancer? Eur J Cardiothorac Surg. 2005;27:680-5.

13. Flores RM, Nicastri D, Bauer $T$, et al. Computed tomography screening for lung Cancer: mediastinal lymph node resection in stage IA nonsmall cell lung Cancer manifesting as subsolid and solid nodules. Ann Surg. 2017;265: 1025-33.

14. Murgu SD. Diagnosing and staging lung cancer involving the mediastinum. Chest. 2015;147:1401-12.
15. Stamatis G. Staging of lung cancer: the role of noninvasive, minimally invasive and invasive techniques. Eur Respir J. 2015:46:521-31.

16. Koike T, Yamato Y, Yoshiya K, Toyabe S. Predictive risk factors for mediastinal lymph node metastasis in clinical stage IA non-small-cell lung cancer patients. J Thorac Oncol. 2012;7:1246-51.

17. Gao SJ, Kim AW, Puchalski JT, et al. Indications for invasive mediastinal staging in patients with early non-small cell lung cancer staged with PETCT. Lung Cancer. 2017;109:36-41.

18. Gómez-Caro A, Boada M, Cabañas M, et al. False-negative rate after positron emission tomography/computer tomography scan for mediastinal staging in cl stage non-small-cell lung cancer. Eur J Cardiothorac Surg. 2012;42:93-100.

19. Wang J, Welch K, Wang L. Negative predictive value of positron emission tomography and computed tomography for stage T1-2N0 non-small-cell lung cancer: a meta-analysis. Clin Lung Cancer. 2012;13:81-9.

20. Lee PC, Port JL, Korst RJ, Liss Y, Meherally DN, Altorki NK. Risk factors for occult mediastinal metastases in clinical stage I non-small cell lung cancer. Ann Thorac Surg. 2007:84:177-81.
Ready to submit your research? Choose BMC and benefit from:

- fast, convenient online submission

- thorough peer review by experienced researchers in your field

- rapid publication on acceptance

- support for research data, including large and complex data types

- gold Open Access which fosters wider collaboration and increased citations

- maximum visibility for your research: over $100 \mathrm{M}$ website views per year

At $\mathrm{BMC}$, research is always in progress.

Learn more biomedcentral.com/submissions 